\title{
Cue enhancement as a function of task set
}

WALTER C. GOGEL

UNIVERSITY OF CALIFORNIA, SANTA BARBARA

The hypothesis was tested that the intention to use a particular cue relation would enhance the effectiveness of that particular cue in determining the resulting perception. For this purpose, a situation was presented in which the apparent depth position of an object in a configuration of objects would differ depending upon which of two possible cue relations (size cues) were used. The results support the conclusion that the perceived depth position of the object differed in the expected directions as a function of the task set. The data of the study are discussed with respect to the "adjacency principle" which states that cue efficiency is determined by the relative adjacency of objects between which the cues occur. Although the effect of cue set upon the perception seems to be small compared with that of adjacency, it cannot completely be ignored.

It is clear that set can modify behavior. The importance and role of set in the modification of perception, however, is less clear. It seems that set can influence perception by the process of emphasizing or de-emphasizing certain aspects or parts of the stimuli. For example, the set to perceive the number of letters in a tachistoscopic presentation may prevent the perception of their color, or the set to perceive a hidden figure may increase the likelihood that it will be noticed (Gibson, 1941). Thus, set probably has a selective role in perception. But, whether set also can change the perceived characteristics of the objects is more in doubt (cf., Allport, 1955; Haber, 1966). It is sometimes considered, for example, that set or attitude can modify perceived extents. In a number of instances in which it is asserted that such effects occur it is not obvious why the resulting perceptual modifications should be in one direction rather than another. It is not obvious, for example, why an increase in the value of an object should make it appear larger (cf., Dukes \& Bevan, 1952; Bruner \& Postman, 1948), or why in an Ames distorted room a marital partner should appear more normal in size than a stranger (cf., Wittreich, 1961). However, a rationale as to the direction in which set or intention can affect perceived extent under certain circumstances is avallable as a consequence of the selective role of set in perception. Task set could result in the enhancement or increased effectiveness of a particular cue involved, for example, in determining a perceived depth. If the increased effectiveness of this cue results in a change in perceived depth, it follows that the perceptual change could be attributed to task set.

The purpose of the present experiment was to provide a test of the above rationale for expecting task set (intention) to modify perceived extent. A previous experiment (Gogel, 1963) has suggested that under appropriate conditions a perceived depth could be modified somewhat by the intention of the observer $(O)$. The appropriate conditions consisted of a visual situation in which a familiar object would appear at different depth positions depending upon the relative dominance of depth cues occurring between it and two other objects. Similar conditions were used in the present study except that the stimuli were especially arranged to provide a more sensitive test of the perceptual consequences of task set.

\section{Method}

Front view schematic diagrams of the three visual displays used in the present experiment are shown in Fig. 1. Objects $A, B$, and $D$ were positive transparencies of seven-of-spades playing cards. Object B was twice the size of a normal playing card $(11.4 \times 17.8 \mathrm{~cm})$ and Objects $\mathrm{A}$ and $\mathrm{D}$ were the size of a normal playing card $(5.7 \times 8.9 \mathrm{~cm})$. Object $\mathrm{C}$ was formed from a square of neutral density film $(17.8 \mathrm{~cm}$ on a side). All four objects were transilluminated with white light by mounting them in front of a diffused source (light box). All were viewed monocularly (with the right eye only), and all were at the same distance from $O$ $(305 \mathrm{~cm})$. As is indicated in Fig. 1, Object $\mathrm{C}$ appeared to overlay Card $B$ and to be overlaid by Card $D$. This effect was produced by cutting away $22.4 \%$ of the upper right area of Card $B$ and $4 \%$ of the upper right area of Card C, and carefully fitting the objects together. The positions of the cards in each of the displays was always the same. The position of Card A was $15 \mathrm{~cm}$ to the right of Card $B$ and $15 \mathrm{~cm}$ below Card $D$. The luminance of the playing cards and Object $C$ was 3.0 and $0.5 \mathrm{ft}-\mathrm{L}$, respectively, as measured from the position of O's eye. Only the objects shown in Fig. 1 were visible, with the remainder of the field of view in darkness. Objects $A, B$, and $D$ appeared to $O$ as seven-of-spades playing cards and Object $C$ appeared

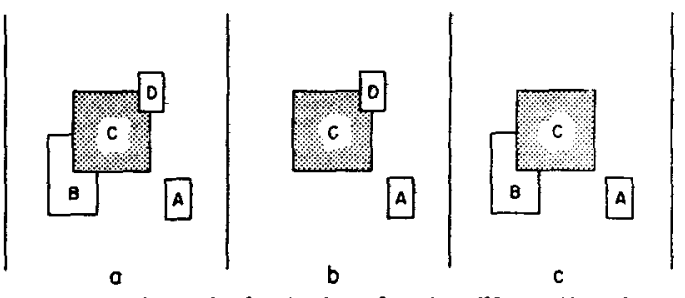

Fig. 1. A schematic front view drawing illustrating the positions of the normal-sized cards ( $A$ or $D$ ), the double-sized card $(B)$, and the gray square $(C)$ for the condition in which all three cards were present (Fig. 1a) or in which one card was absent (Fig. 1b or Fig. 1c). 
to be a gray square. Only one of the displays in Fig. 1 was presented at any one time. Two light boxes were used in producing the displays. One light box was used to transilluminate the displays of Fig. $1 \mathrm{a}$ and $1 \mathrm{~b}$, with the latter display being produced from the former by carefully covering Card B. A separate light box was used for the display of Fig. 1c. A partially transmitting-partially reflecting mirror permitted the display of Fig. 1c to be presented at the same optical distance and direction as the displays of Fig. 1a or $1 \mathrm{~b}$.

The viewing position of $O$ was enclosed with black velveteen and contained a head and chin rest and a viewing aperture. A light adaptation surface ( $3 \mathrm{ft}-\mathrm{L}$ ) located to the left of $O$ 's position could be turned on or off by the experimenter (E) for the purpose of light adapting $O$. An apparatus for measuring perceived depth was also located at the position of $O$. It consisted of two vertical rods positioned at about the level of $O$ 's waist with the right rod movable laterally. $O$ grasped the rods, one in each hand, and adjusted the lateral distance between the rods until this distance seemed to be equal to the perceived depth between the designated playing cards. The rods were invisible to $O$, with the hand adjustments being made kinesthetically. An attached centimeter ruler permitted E to note the adjusted separation of the rods. During the judgments, the interior of the viewing position was completely dark, i.e., no objects or surfaces were visible to $O$ except the objects of the display.

Twenty men served as Os. Each $O$ had a visual acuity in his right eye (corrected if necessary) of at least 20/20. There were five experimental tasks:

Task 1-to indicate the perceived depth between Cards A and D with Card B present (Fig. 1a);

Task 2-to indicate the perceived depth between Cards A and D with Card B absent (Fig. 1b);

Task 3-to indicate the perceived depth between Cards A and B with Card D absent (Fig. 1c);

Task 4-to indicate the perceived depth between Cards A and B with Card D present (Fig. 1a);

Task 5-to indicate the perceived depth between Cards B and D (Fig. 1a).

Each $O$ made three judgments of the depth between two designated playing cards and, following this, faced the light adaptation surface which was turned on for approximately $10 \mathrm{sec}$. This procedure was repeated for each of the five tasks. The order in which the five tasks were completed was systematically varied between Os. Prior to participating in the experiment, the Os were carefully instructed to judge only the depth between the cards. A small pegboard with two pegs was used to illustrate the difference between frontoparallel and depth extents. Following the experiment, the Os were questioned to be certain that the extent which was indicated by the hand adjustment reflected the perceived depth (not the frontoparallel separation) between the cards.
The basic design of the experiment can be discussed with the aid of Fig. 1a. In Fig. 1a, Card D which was retinally smaller than Card B would appear to be more distant than Card B were it not for the conflicting interposition cue introduced by Object $C$. The interposition cue from object $C$ caused Card D to appear less distant than Card $B$ in spite of the relative size cue between these cards. Under these circumstances, consider the perceived relative depth position of Card A. According to the size cue between Cards A and D, Card A should appear at the distance of Card D, i.e., in front of Card B. But, according to the size cue between Cards $A$ and $B$, Card $A$ should appear behind Card B, i.e., also behind Card D. Thus, the apparent depth position of Card $A$ in the configuration of objects would depend upon whether the size cue between Cards $A$ and $\mathrm{D}$ or between Cards $\mathrm{A}$ and $\mathrm{B}$ is the stronger. If task set or intention enhances the effectiveness of a size cue, Card A should appear to be more distant in the configuration when the task is to judge its depth position with respect to Card $B$ rather than with respect to Card $D$. Whether or not this result will occur constitutes a test of the ability of task set to modify a perceived depth by means of the intermediary process of cue enhancement.

Two controls were used. These are illustrated by the displays of Fig. 1b and 1c. If $O$ used the size cues to depth appropriately, Card A should have appeared approximately at the distance of Card $D$ in the display of Fig. 1b, and behind Card $B$ in the display of Fig. 1c. It was required, therefore, that $O$ perceive Card $A$ as more distant than Card $B$ in the display illustrated in Fig. 1c in order for the results from that $O$ to be used in the study. It was also required that the interposition cue be effective, i.e., that Card D should appear in front of Card B in Fig. 1a. The latter requirement insured that Card $A$ would appear at different distances whenever a change occurred in the relative effectiveness of Cards $B$ and $D$ in determining the apparent depth position of Card A.

\section{Expected Results}

The results which would be expected if task set were to modify perceived depth are illustrated in Fig. 2. Figure 2 is a top view schematic drawing indicating the expected apparent positions of the objects shown in the display of Fig. 1a. The prime notation is used to indicate that perceptions are being represented, with the pair of cards being judged shown by the subscripts associated with the perceived depth $d^{\prime}$. Two apparent depth positions of Card $A$ are indicated. The position $A_{\mathfrak{h}}$ is a perceived position of Card $A$ which is expected to occur with the display of Fig. 1a when the task is to judge where Card A appears with respect to Card D. The position $A_{f}^{\prime}$ is a perceived position of Card A which is expected to occur with the display of Fig. 1a when the task is to judge where A 

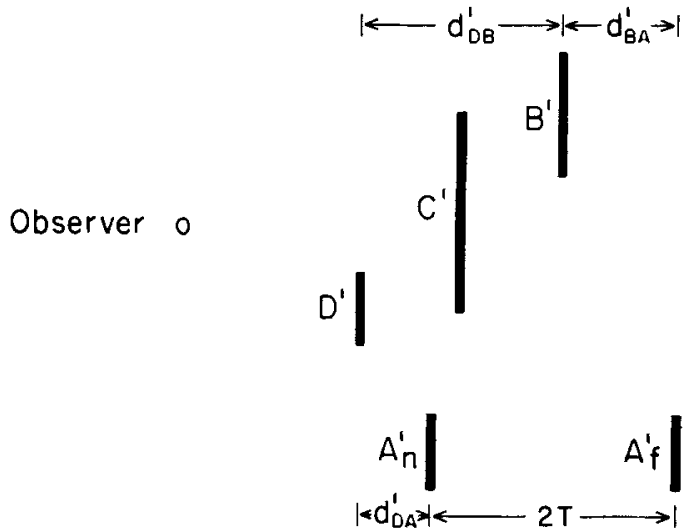

Fig. 2. A schematic top view drawing illustrating the possible enhancement of the effectiveness of size cues as a function of task set.

appears with respect to $B$. If task set is effective, $A_{n}^{\prime}$ rather than $A_{f}^{\prime}$ should be closer to $O$ with this expected difference labeled $2 \mathrm{~T}$. Half this difference (T) can be attributed to the intention of judging the depth position of Card A with respect to one of the other cards. It follows from Fig. 2 that the average value (T) of the effect of task set on perceived depth is

$$
T=\frac{d^{\prime}{ }_{D B}+d_{B A}^{\prime}-d^{\prime}{ }_{D A}}{2}
$$

where $d_{D B}, d_{B A}$, and $d_{D_{A}}$ are the perceived depths between Cards $D$ and $B$, Cards $B$ and $A$, and Cards D and $\mathrm{A}$, respectively.

\section{Obtained Results}

The average results from the relative depth judgments are given in Table 1. Each data entry in Table 1 is an average of 20 scores, one from each $O$ where each score is the mean of three kinesthetic adjustments of the hand apparatus. A positive value in Table 1 indicates that Card $A$ appeared behind the card with which it was being judged and a negative value indicates the converse. According to the scores indicating the perceived depth between Cards $D$ and $B$, the interposition cue resulting from Object $C$ was effective, i.e., D was perceived to be in front of $B$ with an average value of $18.4 \mathrm{~cm}$. However, the scores from 11 Os indicated that Card $A$ was not perceived to be more distant than Card B under the control conditions illustrated in Fig. 1c, 1.e., with Card D absent. Clearly, if A did not appear behind $B$ with $D$ absent, the effect of task set in judging the perceived depth between $B$ and $A$ under the conditions of Fig. 1a (D present) would be difficult to evaluate. Therefore, 11 other Os were substituted in the study for the Os who did not percelve $A$ as more distant than $B$ in the control situation (Fig. 1c). The value of $T$ as computed from the average results obtained from Tasks 1, 4, and 5 using Equation (1) is $8.4 \mathrm{~cm}$. Using the $t$ test $\left(t_{19}=2.74\right)$ this value is significantly different from zero at the .05 level.

\section{Discussion}

As determined by $T$, the average perceived position of $A$ in the configuration of objects was more distant when A was judged with respect to $B$ rather than D. The evidence is, therefore, that the set to judge the depth position of one object with respect to another object can enhance the effectiveness of the depth cue between these objects, as determined in the present experiment by a change in perceived depth.

If the set to use the size cue between Card A and one of the other cards had made this relation completely dominant, the perceived depth between these two cards should have been the same as though the third (extraneous) card had not been present. Thus, the total possible effect of task set, in this experiment, can be computed by substituting in Equation (1), the perceived depth between $\mathrm{A}$ and $\mathrm{D}$ and between $\mathrm{A}$ and $\mathrm{B}$ as determined from the control situation (Task 2, Fig. 1b and Task 3, Fig. 1c) rather than from the experimental situation (Tasks 1 and 4, Fig. 1a). This maximum possible value of $T$ is 24.1 , which is 2.9 times as large as the average value of $T(8.4)$ obtained from the experimental situation. Task set, therefore, while important in the present study, is not equivalent perceptually to removing the extraneous card (the card not involved in the judgment).

In the previous study (Gogel, 1963) evidence was provided for the "adjacency principle" as it relates to size cues to distance. According to the adjacency principle, the perceived depth position of an object in a configuration of objects is mainly determined by whatever cues occur between it and adjacent (not displaced) objects. In that experiment, Card A was to the left of Card B such that it was adjacent to Card B and displaced from Card D. It was found that, although the effect of Card B on the apparent position of Card A was dominant, some small effect could be attributed both to Card D and to task set. In the present experiment, A was intentionally equally separated from B and from D. A method of calculating the effect of Card B and of Card D upon the apparent position of

Table 1. The Average Perceived Depth in Centimeters Between Playing Cards as Measured by the Hand Adjustments

\begin{tabular}{|c|c|c|c|}
\hline $\begin{array}{l}\text { Presence or } \\
\text { Absence of } B\end{array}$ & $\begin{array}{c}\text { Perceived Depth } \\
\text { of } A \text { Behind D }\end{array}$ & $\begin{array}{l}\text { Presence or } \\
\text { Absence of D }\end{array}$ & $\begin{array}{c}\text { Perceived Depth } \\
\text { of A Behind B }\end{array}$ \\
\hline B Presest & & D Absen $\dagger$ & \\
\hline $\begin{array}{l}\text { (Tosk 1) } \\
\text { B Absent }\end{array}$ & $+13.5 * *$ & $\begin{array}{l}\text { (Task 3) } \\
\text { D Present }\end{array}$ & $+25.2 * *$ \\
\hline $\begin{array}{l}\text { (Task 2) } \\
\text { Diff. }\end{array}$ & $\begin{array}{l}-4.5 \\
18.0 * *\end{array}$ & $\begin{array}{l}\text { (Task 4) } \\
\text { Diff. }\end{array}$ & $\begin{array}{l}+12.0 \\
13.2 *\end{array}$ \\
\hline
\end{tabular}

$* p<.05$ (two-tailed $t$ test. $d f=19$ )

** $p<.01$ (two-tailed test, $d f=19$ ) 
Card A (Gogel, 1963) can be applied to the present experiment. This method determines the effect of Card B by subtracting the depth perceived between Card A and Card D obtained when B is present (Task 1 ) from that obtained when $B$ is absent (Task 2). Similarly, the effect of Card D is determined by subtracting the perceived depth between Card A and Card B obtained when $D$ is absent (Task 3 ) from that obtained when $D$ is present (Task 4). The results are that $18.0 \mathrm{~cm}$ and $13.2 \mathrm{~cm}$ of depth (see Table 1) can be attributed to Cards $B$ and $D$, respectively, with the difference between these values not significant at the .05 level $\left(t_{19}=.61\right)$. It can be concluded in agreement with the adjacency principle, that placing Card A equally far from Cards B and D approximately equalizes the effect of Card B and Card D on Card A.

Since the 18.0 and $13.2 \mathrm{~cm}$ values indicate the effects on Card A of the presence of the card not involved in the comparison, they represent the amount by which this extraneous card is more effective than the task set in determining the apparent depth of Card A. The adjacency principle and task set are clearly different and sometimes antagonistic factors. The adjacency principle indicates the relative strengths of cues between objects on the basis of their adjacency while the effect of set is considered without regard to the relative distance between the objects. Even under the balanced separations of the present experiment in which the effect of task set would be expected to be maximal, it seems that the effects involved in the adjacency principle were stronger than those of the task set.

It remains to be considered why one-third of the Os tested in the present study failed to meet the control requirement that Card $A$ be perceived as more distant than Card B when Card D was absent (Fig. 1c). This result can be understood in terms of the equidistance tendency (Gogel, 1965) between Objects A and C. The equidistance tendency states that in the absence of strong distance cues, there is a tendency for objects to appear equidistant, with the strength of this tendency being inversely related to directional separation. It will be seen in Fig. 1c that Object $C$ which appears in front of $B$ is directionally close to A. Thus, the size cue to distance which would make A appear behind $\mathrm{B}$ is opposed by the equidistance tendency which would make $A$ appear at the distance of $C$, i.e., in front of B. It has been demonstrated under somewhat similar conditions that the equidistance tendency can modify the perceived depth from relative size cues (Gogel, 1956, Experiment IV). Therefore, it is likely that even those Os who perceived Card A to be behind Card $B$ in the control condition did not see A as being as far behind $B$ as would be expected from the relative size cue in the absence of Object $C$.

\section{Conclusions}

The present experiment demonstrates that under certain conditions the task set or intention of the $O$ can modify the perceived depth between objects resulting from the relative size cue. This effect is assumed to occur as a consequence of the cue enhancement resulting from the intention to directly compare the particular objects. However, the effect of task set, while significant in this experiment, must be considered as minor compared with the influence of surrounding objects not directly involved in the judgment. A comparison of the results from the present and a previous experiment supports the position that the relative effectiveness of cues depends upon the relative separation of the objects (the adjacency principle).

\section{References}

Allport, F. Theories of perception and the concept of structure. New York: John Wiley \& Sons, 1955.

Bruner, J. S., \& Postman, L. Symbolic value as an organizing factor in perception. J. soc. Psychol., 1948, 27, 203-208.

Dukes, W. F., \& Bevan, W., Jr. Size estimation and monetary value: A correlation. $J$. Psychol, 1952, 34, 43-53.

Gibson, J. J. A critical review of the concept of set in contemporary experimental psychology. Psychol. Bull., 1941, 38, No. 9.

Gogel, W. C. The tendency to see objects as equidistant and its inverse relation to lateral separation. Psychol. Monogr., 1956 , 70 , No. 4 (Whole No. 411), 1-17.

Gogel, W. C. Size cues and the adjacency principles. CARI Report No. 63-28, 1963, Oklahoma City, Oklahoma.

Gogel, W. C. The equidistance tendency and its consequences. Psychol. Rev., 1965, 64, 153-163.

Haber, R. N. The nature of the effect of set on perception. Psychol. Rev., 1966, 73, 335-350.

Wittreich, W. F. The Honi phenomenon: A case of selective perceptual distortion. In F. P. Kilpatrick (Ed.), Explorations in transactional psychology. New York: New York University Press, 1961.

\section{Note}

1. This study was conducted at the Civil Aeromedical Institute, Oklahoma City, Oklahoma. The author wishes to thank Henry W. Mertens and James $C$. Hester for their assistance in collecting and analyzing the data of this study.

(Accepted for publication June 6, 1967.) 